\title{
ESTUDIO DE PERIODICIDADES DE LA PRECIPITACIÓN DE VERANO EN EL VALLE DEL EBRO DESDE EL SIGLO XV ${ }^{1}$
}

\author{
Miguel Ángel SAZ SÁNCHEZ y José CREUS NOVAU \\ Instituto Pirenaico de Ecología \\ Consejo Superior de Investigaciones Científicas, Jaca (Huesca)
}

\begin{abstract}
Resumen: Utilizando la metodología del análisis espectral, se estudia la presencia de algún tipo de patrón cíclico en las series dendroclimáticas de la precipitación de verano de cuatro observatorios del valle del Ebro durante el periodo 1495-1989. Una vez filtrados los datos con objeto de eliminar la variabilidad asociada a la alta frecuencia, han podido identificarse algunos ciclos significativos, destacando uno de en torno a 24 años localizado en tres de los cuatro puntos analizados y que podemos relacionar con el ciclo de Hale. Sólo el observatorio situado en el Pirineo Oriental, a más de $1400 \mathrm{~m}$ de altitud, no presenta frecuencias significativas coincidentes con ese ciclo.

Palabras clave: Análisis espectral, Dendroclimatología, valle del Ebro, ciclo de Hale.

Abstract: A cyclic behaviour in the summer rainfall dendroclimatic time series (14951989) of four weather stations located in the Ebro Valley is studied using the spectral analysis. Once variability associated to high frequency was removed using a three years moving average filter, a 24 year cycle related Hale cycle was identified in three data base. The weather station located in eastern Pyrennees —above $1400 \mathrm{~m}$ - does not display coincident significant frequences with that cycle.
\end{abstract}

Key words: Spectral analysis, Dendroclimatology, Ebro Valley, Hale cycle.

\section{INTRODUCCIÓN}

La disyuntiva entre la presencia de algún tipo de patrón cíclico en el comportamiento de las variables climáticas, o el que éstas respondan únicamente a la evolución propia de

\footnotetext{
${ }^{1}$ Este trabajo forma parte del proyecto de investigación CLI96-1862, financiado por la CICYT.
} 
un sistema caótico, es un tema que siempre ha interesado a la comunidad científica en general y de manera especial a climatólogos y meteorólogos.

En la actualidad este debate dista mucho de poderse considerar como cerrado, y si para todos es obvia la existencia de los ciclos diario, estacional y anual, determinados por la posición relativa de la Tierra respecto al Sol, la cuestión no está ni mucho menos clara cuando se especula sobre la posibilidad de que puedan hallarse ciclos en el clima de longitud superior a la anual o no directamente vinculados con los previsibles mecanismos de rotación y traslación.

Lo cierto es que sea cual sea la longitud temporal de los datos analizados y la fuente de información climática utilizada, desde la instrumental a la variada información proxy, fácilmente se comprueba que las variables climáticas (en especial precipitación y temperatura), lejos de mantenerse en valores cercanos a los de los promedios que utilizamos para caracterizarlas, muestran oscilaciones por encima y por debajo de la media. Momentos más fríos o cálidos y secos o húmedos, aparecen en cualquiera de las escalas temporales analizadas, desde días o semanas a décadas, siglos e incluso milenios, invitándonos a pensar en la existencia de algún tipo de periodicidad en esos datos.

En este sentido se han multiplicado en los últimos decenios los trabajos dedicados por un lado a la búsqueda de alguna posible señal cíclica subyacente en las bases de datos climáticos y por otro a la explicación en términos físicos de los comportamientos periódicos que de forma más frecuente hayan ofrecido significación estadística.

Los resultados obtenidos han sido en ocasiones importantes. Por mencionar algunos de ellos (BURROUGHS, 1992), puede señalarse la confirmación de la relación existente entre el fenómeno de El Niño y variaciones climáticas a escala global, con periodicidades de 5 a 7 años; el descubrimiento de una inversión periódica en la dirección de los vientos en la estratosfera, que acaece cada 27 meses y que algunos relacionan con la oscilación bianual descrita en muchas series de datos; o la verificación, gracias a mediciones de satélite, de que la energía emitida por el sol sufre variaciones de alrededor de un $0,1 \%$ en función del mayor o menor número de manchas solares en su superficie, con una periodicidad media de 11 años.

Estos resultados han animado a los investigadores a continuar con la labor de identificación de las conexiones existentes entre determinados fenómenos físicos y la presencia de comportamientos cíclicos en el clima. Pero la respuesta del clima frente a los procesos físicos nunca sucede de forma lineal (ROYER et al, 1994) y el complejo cúmulo de interacciones que se produce entre los distintos componentes del sistema climático dificulta la interpretación de la relación existente entre mecanismos físicos y clima.

La dendroclimatología también se vio envuelta prácticamente desde sus inicios en el debate sobre la presencia o no de ciclos dentro de las series climáticas generadas a partir de sus estudios. Ya en el primer tercio del siglo XX, Douglass describió la relación 
existente entre el crecimiento de los árboles en el entorno del Mar Báltico y los ciclos de manchas solares (DOUGLASS, 1933). Pero tampoco faltaron por aquel entonces trabajos que llegaban a conclusiones radicalmente opuestas, destacándose precisamente la no presencia de ese comportamiento cíclico en datos dendroclimáticos (BONNER, 1935).

Como en otras ciencias vinculadas con la climatología, a partir de los años setenta aumentó considerablemente dentro de la dendroclimatología el número de estos trabajos. Algunos de los más relevantes aparecen citados en la bibliografía (LAMB, 1972; LA MARCHE, 1974; MITCHELL et al, 1979; BABOS, 1986; CURRIE, 1991a, b y c; CLAEVELAND et al, 1992; CURRIE, 1992; MITRA et al, 1992, CHAMBERS et al 1999). En las series analizadas se comprobaba la significación estadística de varios ciclos de distinta duración (10-12 años, 18-20 años, 22-23 años, 30 años, 90 años, 100 años, 200 años) y que en general, en el caso de ciclos de amplitudes dentro del rango de los 2 a los 100 años, coincidían con los detectados a partir de información instrumental, pero con la ventaja, en términos estadísticos, de haberse descrito a partir de series de datos mucho más largas.

En España los trabajos en este sentido han sido relativamente escasos, y sólo ocasionalmente, como una parte más del estudio temporal de las series reconstruidas, aparece algún análisis encaminado hacia la detección de posibles periodicidades dentro de las series dendroclimáticas (CREUS et al, 1980; CREUS et al, 1995; CREUS, 1996, 1998).

En el caso que nos ocupa va a analizarse la posible presencia de algún tipo de patrón cíclico en la precipitación del tercer trimestre (julio, agosto y septiembre) en el valle del Ebro a lo largo de los últimos cinco siglos.

Durante ese periodo la sucesión de sequías de distinta duración parece haber sido un fenómeno habitual en este espacio geográfico (SAZ et al, 1998) y su mayor o menor intensidad puede depender en muchas ocasiones de los totales de precipitación de verano. Aunque la identificación de algún tipo de comportamiento cíclico en esta variable en ningún modo ha de ser considerada como una herramienta válida para realizar predicciones sobre el advenimiento de fases secas o húmedas, sí contribuirá sin duda el mejor conocimiento de la complicada pluviometría estival de esta zona peninsular.

\section{DATOS Y METODOLOGÍA}

Los datos utilizados para este trabajo son las series dendroclimáticas de la precipitación total del tercer trimestre, reconstruida para el periodo 1495-1989 en cuatro observatorios del valle del Ebro: Haro (La Rioja), Pallaruelo de Monegros (Huesca), Capdella (Lérida) y Vimbodí (Tarragona), cuya localización, coordenadas y altitud aparecen en el mapa 1 y tabla I. 

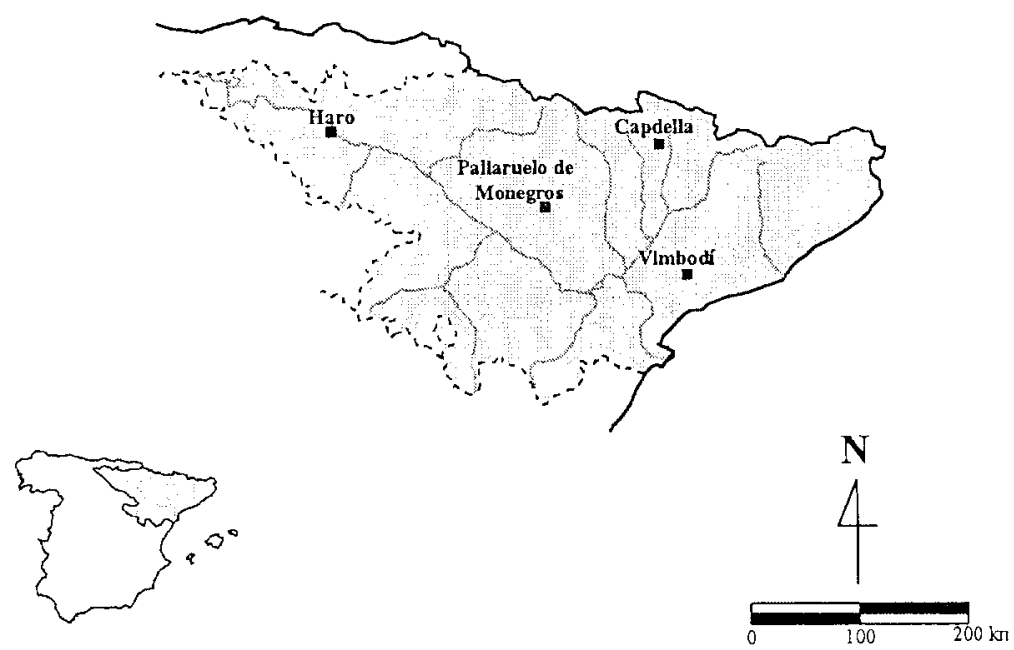

Mapa 1. Localización de los observatorios reconstruidos en el cuadrante nororiental español

Tabla 1. Altitud sobre el nivel del mar y coordenadas de los observatorios

\begin{tabular}{|lcc|}
\hline & Altitud & Coordenadas \\
\hline Haro & $479 \mathrm{~m}$ & $02^{\circ}-51^{\prime}-07^{\prime \prime} \mathrm{W} ; 42^{\circ}-34^{\prime}-30^{\prime \prime} \mathrm{N}$ \\
Pallaruelo & $356 \mathrm{~m}$ & $00^{\circ}-12^{\prime}-41^{\prime \prime} \mathrm{W} ; 41^{\circ}-42^{\prime}-17^{\prime \prime} \mathrm{N}$ \\
Capdella & $1422 \mathrm{~m}$ & $00^{\circ}-59^{\prime}-28^{\prime \prime} \mathrm{E} ; 42^{\circ}-27^{\prime}-55^{\prime \prime} \mathrm{N}$ \\
Vimbodí & $487 \mathrm{~m}$ & $01^{\circ}-02^{\prime}-28^{\prime \prime} \mathrm{E} ; 41^{\circ}-22^{\prime}-20^{\prime \prime} \mathrm{N}$ \\
\hline
\end{tabular}

Con la información trimestral y anual de la precipitación y temperatura, reconstruidas en estos observatorios hasta el siglo XV mediante la metodología dendroclimática, ya se han publicado algunos resultados referidos a su evolución temporal (CREUS, 1991, 1996; CREUS et al 1999; SAZ et al 1998, 1999).

La técnica utilizada para rastrear en estas series de datos alguna posible componente cíclica, ha sido el análisis espectral. Esta metodología deriva del más conocido análisis armónico o de Fourier, si bien se muestra más eficaz en el caso de estudiar en las series de tiempo componentes periódicas de las que no se tienen referencias previas respecto a su probable amplitud (ESSENWANGER, 1986).

Previamente a la aplicación del análisis espectral a cada una de las series de tiempo, es aconsejable evaluar la existencia en ellas de tendencias a largo plazo que pudiesen distorsionar los resultados (MANLEY, 1974). Sólo en el caso de Capdella fue necesario eliminar la tendencia detectada en su serie de precipitación de verano. En el resto, ninguna de las 
tendencias detectadas alcanzó un nivel de significación igual o superior al $90 \%$, por lo que no fueron eliminadas.

La base matemática que subyace en el análisis espectral, es que cualquier función matemática puede ser descompuesta en un conjunto de frecuencias fundamentales periódicas, o lo que es lo mismo, en armónicos de diferente amplitud cuyo número, al tratarse de series de datos discretos (como es nuestro caso) es igual a la mitad del número de datos u observaciones. La amplitud de estos armónicos también es conocida, siendo en el primero de ellos (denominado en ocasiones fundamental) igual al número de datos de la serie $(\mathrm{N})$, en el segundo igual a $\mathrm{N} / 2$, en el tercero $\mathrm{N} / 3$, en el cuarto $\mathrm{N} / 4$, etc. Cada uno de estos armónicos o frecuencias fundamentales recogerá una parte de la varianza total de la serie (ESSENWANGER, 1986).

La presentación más usual del análisis espectral se realiza mediante un periodograma, donde se muestra gráficamente en qué proporción contribuyen cada uno de los armónicos a la varianza total de la serie, es decir, qué cantidad de la varianza está asociada a cada una de las frecuencias del periodograma.

Los picos del periodograma pueden ser indicativos de posibles periodicidades en los datos. Para distinguir lo aleatorio, resultado del mero azar estadístico, de lo que no lo es, ha de aplicarse algún tipo de test. Para ello se ha utilizado el test de Fisher o del estadístico G (DAVIS, 1986, SWAN et al, 1995), basado en la relación existente entre la máxima varianza asociada a una determinada frecuencia $\left(\mathrm{s}^{2}{ }_{\text {máx }}\right)$ y la varianza total de la serie $\left(\mathrm{s}^{2}\right)$ :

$$
\mathrm{G}=\mathrm{s}_{\text {máx }}^{2} / 2 \mathrm{~s}^{2}
$$

Para que el pico sea significativo, el valor $G$ obtenido debe ser igual o mayor al valor crítico Gc, cuyo cálculo se realiza según la siguiente fórmula:

$$
\mathrm{Gc}=1-\mathrm{e}^{(\ln \mathrm{p}-\mathrm{hum}) / \mathrm{n}-1}
$$

siendo lnp el logaritmo neperiano de el nivel de probabilidad elegido y $\mathrm{m}$ la mitad del número total de datos de la serie (para series con más de 30 datos).

\section{RESULTADOS OBTENIDOS A PARTIR DE LOS DATOS ORIGINALES}

Los periodogramas resultado de aplicar el análisis espectral a cada una de las series de datos originales (Figuras 1 a 4), muestran un único pico señalado en las series de Haro y Capdella y la presencia de un número mayor de picos espectrales destacables en Pallaruelo y Vimbodí.

En Haro (Figura 1), este pico más destacado se corresponde con una frecuencia de $0,0424 \mathrm{cpa}$ (ciclos por año), que recoge un 7,64\% de la varianza total de la serie. Tras este 
pico principal, aunque con una aportación a la varianza total sensiblemente inferior, destaca el de los 0,0606 cpa, con una proporción de la varianza total del 3,12\%. El resto de picos espectrales quedan ya porcentualmente muy lejos de los dos señalados, en especial los que encontramos más allá de los 0,3 cpa (ciclicidad de alta frecuencia), donde la participación en la varianza total de la serie es casi nula, quedando ésta concentrada, fundamentalmente, en un rango que va de los 0,03 a los 0,07 cpa.

El caso de Pallaruelo es diferente y es mayor dentro del periodograma el número de picos espectrales destacados (Figura 2). El más importante de ellos es el de los 0,0566 cpa, que concentra un 3,52\% de la varianza total de la serie. Muy cerca queda el pico inmediatamente anterior a éste, $0,0545 \mathrm{cpa}$, con un 3,19\% de varianza asociada. Entre los 0,04 y $0,06 \mathrm{cpa}$, aparecen varias frecuencias importantes, característica que en cierto modo comparte con el observatorio anterior, si bien en el del sector central de la Depresión del Ebro

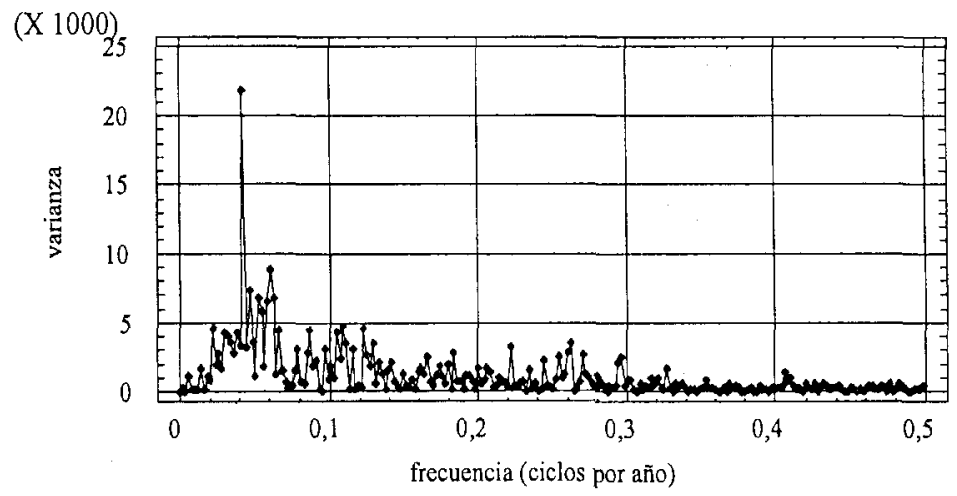

Figura 1. Periodograma de la precipitación total de verano en Haro (1495-1989)

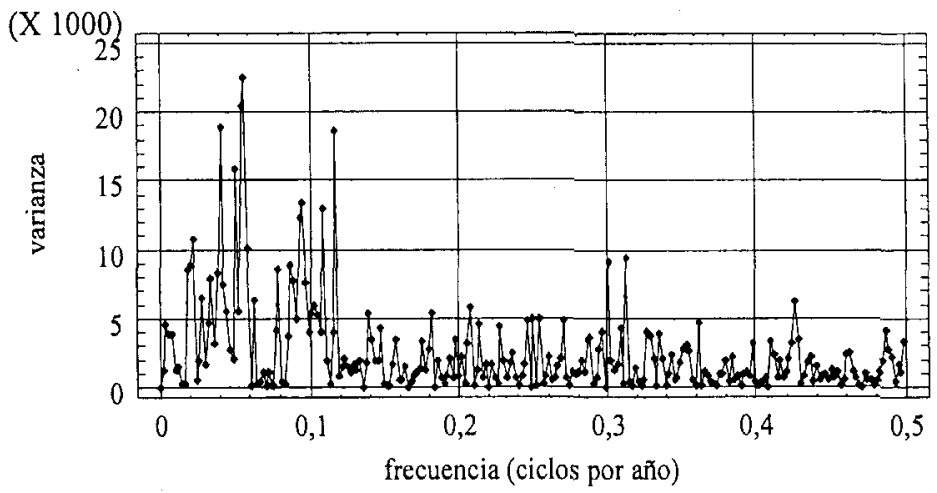

Figura 2. Periodograma de la precipitación total de verano en Pallaruelo (1495-1989) 
encontramos un nuevo grupo de frecuencias con destacados porcentajes de varianza asociada en torno a los 0,09 y 0,12 cpa, como es el pico correspondiente a los 0,1172 cpa, que recoge un $2,89 \%$ de la varianza total. La ciclicidad de alta frecuencia, dentro de no ser demasiado reseñable, adquiere en este observatorio una importancia relativamente mayor a la que hemos visto en Haro, destacando a partir de los 0,3 cpa dos picos: el de 0,301 cpa, con un 1,41\% de varianza asociada y el de $0,3131 \mathrm{cpa}$, con un $1,41 \%$.

De nuevo encontramos un único pico espectral claramente destacado en el periodograma de la serie de Capdella (Figura 3), que en este observatorio pirenaico se corresponde con una ciclicidad de más baja frecuencia que en los casos anteriores, de 0,0121 cpa, siendo el porcentaje de la varianza asociada del $4,24 \%$. El resto quedarían bastante lejos de este pico principal, destacando únicamente las frecuencias de 0,0566 y $0,0323 \mathrm{cpa}$, con porcentajes asociados del $2,12 \%$ y $2,07 \%$.

En el periodograma de Vimbodí vemos que, como en Pallaruelo, existen varios picos espectrales destacados, concentrados ahora entre los 0,02 y $0,07 \mathrm{cpa}$, no apareciendo ese segundo

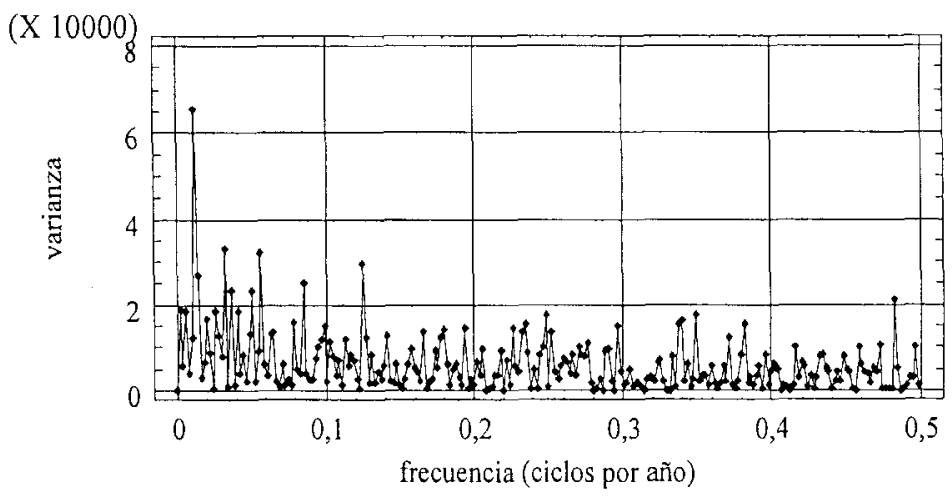

Figura 3. Periodograma de la precipitación total de verano en Capdella (1495-1989)

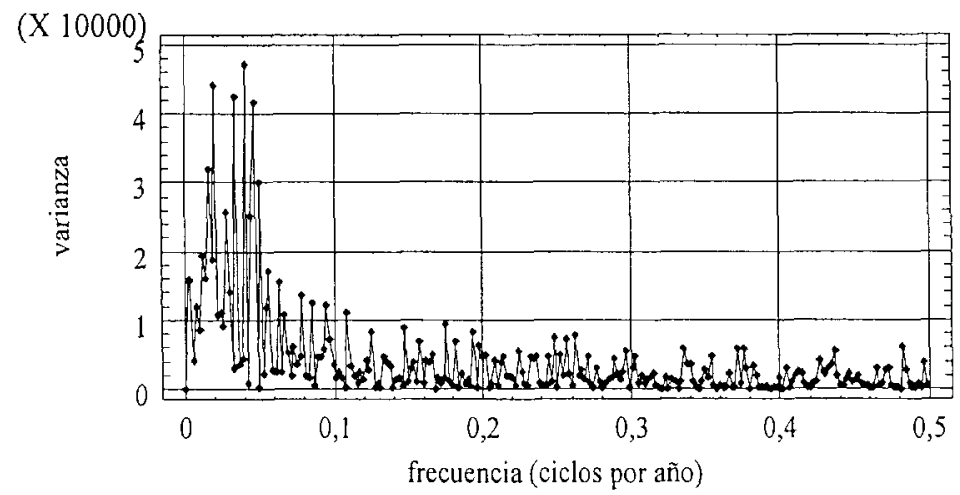

Figura 4. Periodograma de la precipitación total de verano en Vimbodí (1495-1989) 
grupo de frecuencias destacadas en tomo a los 0,1 cpa. El pico más importante es el correspondiente a los 0,0404 cpa, que recoge un porcentaje de varianza del 4,58\%, quedando muy cerca el de los 0,0202 , con un 4,26\%. Dentro del periodograma son también reseñables las frecuencias de $0,0343 \mathrm{cpa}$, con un 4,1\%, y 0,0465 cpa, con un 4,04\%. A partir de allí los porcentajes de varianza asociada a cada pico son mínimos, quedando generalmente muy por debajo del $1 \%$.

A cada uno de estos picos más destacados le ha sido aplicado el test de Fisher con objeto de comprobar su significación (ver Tabla 2). El resultado es que sólo en el periodograma de Haro el pico espectral más importante ha podido identificarse como significativo, concretamente el de los 0,0424 cpa, correspondiendo a un ciclo de 23,6 años, cuyo nivel de significación ha superado el $95 \%$. En el resto de observatorios las frecuencias más importantes de los periodogramas no han alcanzado en ningún caso niveles de significación superiores al $90 \%$.

Tabla 2. Resultados del test de Fisher aplicado a los periodogramas de la precipitación de verano en los observatorios reconstruidos en el valle del Ebro

\begin{tabular}{|lcccc|}
\hline & Haro & Pallaruelo & Capdella & Vimbodí \\
& $1495-1989$ & $1495-1989$ & $1495-1989$ & $1495-1989$ \\
\hline Varianza máxima & 21.807 & 22.532 & 65.307 & 47.347 \\
Longitud del ciclo en años & 23,6 & 17,7 & 82,5 & 24,7 \\
Varianza total & 285.601 & 640.375 & 1.527 .840 & 1.034 .230 \\
Número de datos & 495 & 495 & 495 & 495 \\
Significación & $95,0 \%$ & $\mathrm{NO}$ & $\mathrm{NO}$ & $\mathrm{NO}$ \\
\hline
\end{tabular}

\section{RESULTADOS OBTENIDOS A PARTIR DE LOS DATOS FILTRADOS}

El hecho de que en ninguno de los cuatro casos analizados sea importante la proporción de la varianza recogida por los picos espectrales que se encuentran más allá de los 0,3 cpa (con lo que prácticamente habría de rechazarse la posibilidad de que existan en las series ciclos significativos de longitud inferior a los 3,3 años) y que en todos los periodogramas la varianza se concentre fundamentalmente en un rango que va de los 0,01 a los 0,12 cpa, planteó la posibilidad de aplicar algún filtro que eliminase la variabilidad de alta frecuencia, aumentando previsiblemente con ello la proporción de varianza asociada a las frecuencias intermedias. Para ello se utilizó una media móvil de tres años y nuevamente se aplicó el análisis espectral a las series resultantes de aplicar este filtro.

A su vez, también en la nueva serie de Capdella se detectó una tendencia significativa que, como en el caso anterior, fue eliminada antes de realizar el análisis para evitar posibles distorsiones en los resultados finales. 
Los periodogramas resultantes (Figuras 5 a 8) muestran en la baja y media frecuencia, características similares a los anteriores: un único pico destacado en Haro y Capdella y la presencia de un mayor número de ellos en Pallaruelo y Vimbodí.

Se observa que en Haro (Figura 5) el pico espectral más importante se corresponde con la misma frecuencia que en el primer periodograma, la de los 0,0424 cpa, si bien la proporción de varianza asociada es sensiblemente mayor, alcanzando el $11,66 \%$.

En Pallaruelo (Figura 6), sigue manteniéndose como principal el mismo pico que describíamos antes para este observatorio, el de los 0,0567 cpa, en este caso con un 6,6\% de varianza asociada. Pero el segundo pico espectral en importancia ha cambiado, pasando a ser ya no el inmediatamente anterior al señalado, el de los $0,0545 \mathrm{cpa}$, sino el de los 0,0404 cpa, recogiendo un $5,45 \%$ de la varianza.

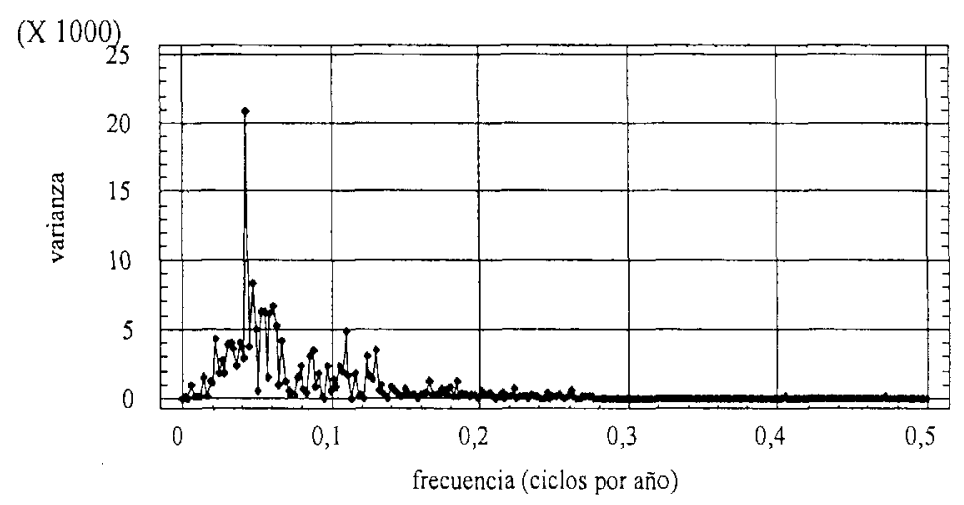

Figura 5. Periodograma de la precipitación total de verano en Haro (1495-1989) filtrada mediante una media móvil de tres años

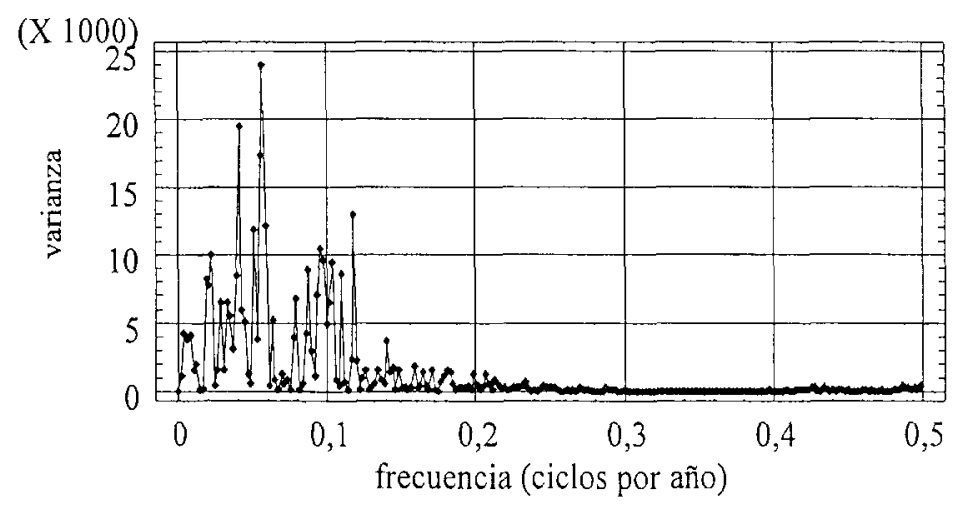

Figura 6. Periodograma de la precipitación total de verano en Pallaruelo (1495-1989) filtrada mediante una media móvil de tres años 
En Capdella sigue manteniéndose un único pico espectral como el más importante dentro del periodograma (Figura 7), coincidente con el detectado en el análisis espectral de los datos originales. Se corresponde con los 0,0121 cpa, duplicando ahora el porcentaje de la varianza asociada, hasta alcanzar el $8,79 \%$.

Y por último, en Vimbodí el primer y segundo picos más importantes (Figura 8) son los mismos que en el periodograma realizado a partir de los datos sin suavizar, incrementándose la proporción de varianza asociada a cada uno de ellos: el de 0,0404 cpa como principal, con un $7,17 \%$ y como segundo el de $0,0202 \mathrm{cpa}$, con un $6,51 \%$.

A cada una de las varianzas asociadas a estos picos espectrales, se les ha aplicado de nuevo el test de Fisher con objeto de comprobar su nivel de significación. Los resultados

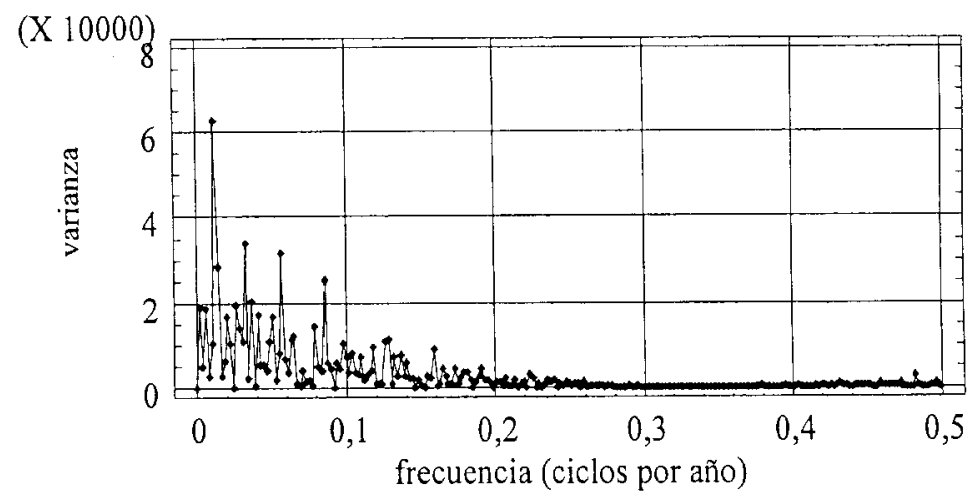

Figura 7. Periodograma de la precipitación total de verano en Capdella (1495-1989) filtrada mediante una media móvil de tres años

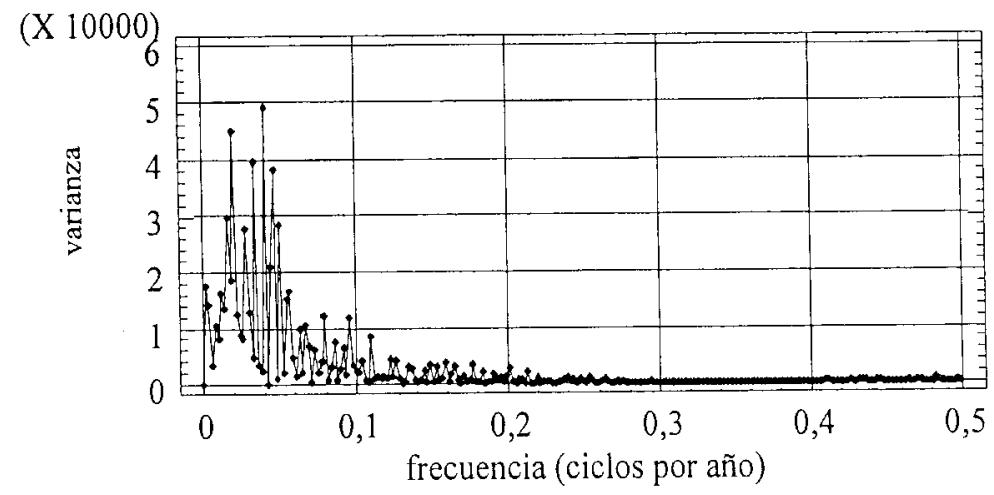

Figura 8. Periodograma de la precipitación total de verano en Vimbodí (1495-1989) filtrada mediante una media móvil de tres años 
obtenidos en esta ocasión son sensiblemente distintos a los vistos para las frecuencias encontradas en los datos originales. Si entonces sólo la frecuencia de los 0,0424 cpa en Haro resultó ser significativa, ahora ha aumentado el número de las que han superado el test de significación (ver Tabla 3).

En Haro sigue apareciendo como significativa la frecuencia de los 0,0424 cpa, asociada a un ciclo de 23,6 años, subiendo en este caso el nivel de significación hasta el 99,9\%. Ningún otro pico espectral del periodograma de Haro ha superado el umbral mínimo del $90 \%$.

El periodograma de Pallaruelo muestra ahora dos frecuencias significativas: al $95 \%$ la de los 0,0567 cpa, correspondiente a un ciclo de 17,7 años, y al $90 \%$ la de los 0,404 cpa, ciclo de una longitud de 24,7 años.

También en Capdella el único pico espectral destacado supera el nivel de significación exigido: la frecuencia de los 0,0121 cpa, que se corresponde con un ciclo de 82,3 años, es ahora significativa al $99 \%$.

Y finalmente en Vimbodí, encontramos que, como en Pallaruelo, existen ahora dos frecuencias que han superado el test de significación: de nuevo aparece la de los 0,0404 cpa, asociada recordemos a un ciclo de 24,7 años, que alcanzaría una significación del $95 \%$, y la de los 0,0202 cpa, asociada a un ciclo de 49,4 años, que supera el umbral del $90 \%$ y que probablemente no es más que un armónico del ciclo de 24,7 años.

Tenemos pues que en tres de las cuatro series analizadas existen periodicidades significativas de 23,6 y 24,7 años. Sólo en los datos de Capdella, observatorio situado en el Pirineo Oriental (en cierto modo en un ámbito climático sensiblemente distinto debido a su elevada altitud), no aparece este mismo comportamiento cíclico, siendo significativa una ciclicidad de baja frecuencia de una amplitud de 82,3 años. En los otros tres observatorios analizados, los ciclos significativos aparecen en ese rango de frecuencias intermedias: en Haro el ciclo

Tabla 3. Resultados del test de Fisher aplicado a los periodogramas de la precipitación de verano, filtrada mediante una media móvil de tres años, en los observatorios reconstruidos en el valle del Ebro

\begin{tabular}{|lcccc|}
\hline & Haro & Pallaruelo & Capdella & Vimbodia \\
& $1495-1989$ & $1495-1989$ & $1495-1989$ & $1495-1989$ \\
\hline Varianza máxima & 20.880 & 22.592 & 62.145 & 48.153 \\
Longitud del ciclo en años & 23,6 & 17,7 & 82,5 & 24,7 \\
Varianza total & 179.041 & 342.399 & 713.804 & 671.595 \\
Número de datos & 495 & 495 & 495 & 495 \\
Significación & $99,90 \%$ & $95,0 \%$ & $99,0 \%$ & $95,0 \%$ \\
Otros ciclos significativos & $\#$ & $24,7(90 \%)$ & $\#$ & $49,4(90 \%)$ \\
\hline
\end{tabular}


de 23,6 años, en Pallaruelo el de 17,7 años como principal pero además el de 24,7 años, ciclo que se repite con esa misma amplitud en Vimbodí, resultando ser también significativo en este observatorio el ciclo de 49,4 años, seguramente armónico del principal.

Ciclos de longitudes dentro del rango de los 20 a 25 años, identificados tanto en series instrumentales como dendroclimáticas, han sido relacionados con el ciclo de Hale, cuya amplitud media es de 22,2 años (LAMB, 1972; BURROUGHS, 1992; CHAMBERS et al, 1999). Una posible explicación física del ciclo de Hale hay que buscarla en mecanismos externos al sistema tierra-atmósfera, concretamente a cambios en la polaridad magnética de las manchas solares que sucederían con esa periodicidad media de algo más de 22 años. Estos cambios no afectarían sólo a la emisión solar, provocando una disminución o un aumento de ésta, sino también a la cantidad de partículas energéticas emitidas por el sol y al propio campo magnético terrestre, variando la cantidad de rayos cósmicos que alcanzan las capas altas atmosféricas y pudiendo llegar a alterar sus propiedades químicas, afectando con ello a todo el sistema climático (BURROUGHS, 1992).

Estas relaciones causa efecto han de tomarse siempre con cierta cautela. Ya se ha mencionado que, dentro del sistema climático, la respuesta a cualquier mecanismo físico no se produce de forma lineal y son muchas y mal conocidas las interacciones que se producen dentro del sistema como para poder atribuir a un único mecanismo cualquier comportamiento periódico detectado en series climáticas. Además los propios procesos estocásticos pueden generar ciclos falsos o armónicos de otros (CREUS, 1996) que por coincidencia en su amplitud relacionemos con los ciclos identificados en procesos físicos conocidos.

Pero independientemente del proceso físico que pueda generarlo, lo cierto es que al menos estadísticamente, sí parece existir un comportamiento periódico de la precipitación de verano en nuestra zona de estudio, con una amplitud media que estaría en torno a los 24 años y del que según parece quedarían excluidas las zonas de alta montaña. Su presencia es evidente en el caso del observatorio más occidental, Haro, y también parece claro que en Vimbodí es el ciclo dominante. En Pallaruelo, si bien ha sido identificado como significativo, queda relegado a un papel secundario, siendo el ciclo principal el de 17,7 años.

\section{CONCLUSIONES}

El estudio de la posible presencia de algún tipo de patrón cíclico en la precipitación de verano en el valle del Ebro, ha mostrado evidencias sobre la existencia de cierto comportamiento periódico de esta variable en los cuatro puntos analizados.

Un primer análisis espectral de las series ofreció como resultado un único ciclo significativo de 23,6 años en Haro, el observatorio más occidental. En el resto de puntos analizados ninguna de las frecuencias del correspondiente periodograma superó el test de significación. 
La periodicidad de alta frecuencia tiene en estas series una presencia prácticamente nula, no llegando en ningún caso a concentrarse una proporción de la varianza importante en las frecuencias existentes en el rango que va de los 0,3 a los 0,5 ciclos por año, quedando así descartada la presencia de ciclos significativos de longitud inferior a los 3 años.

Eliminada toda esa variabilidad relacionada con los ciclos de alta frecuencia después de filtrar las series mediante una media móvil de tres años, en el análisis espectral realizado sobre los datos resultantes se comprobaba que, aunque los periodogramas no variaban su aspecto de forma sustancial en lo que se refiere a la media y baja frecuencia, sí se producía un aumento en la proporción que de la varianza total de cada una de las series absorbían los picos principales. Así en Haro el ciclo de 23,6 años reforzaba su significación, mientras que en Vimbodí y Pallaruelo podían identificarse ciclos significativos de una longitud en ambos casos de 24,7 años. Estos ciclos de amplitudes en torno a los 24 años, podrían relacionarse con el ciclo de Hale, cuya longitud media es de 22,2 años.

El observatorio de Capdella, situado en un ámbito de alta montaña, no comparte este comportamiento cíclico, dominando la baja frecuencia con un ciclo significativo de 82,3 años.

\section{BIBLIOGRAFÍA}

BABOS, K. (1986): «The sunspot activity cycle and the formation of the annual ring width in some wood species». Wood and Fiber 18 (1), 76-83.

BONNER, F.E. (1935): «Climatic cycles in the West: no direct relation found between sunspots, tree rings and rainfall». Civil Engineering, 5, 343-346.

BURROUGHS, W.J. (1992): Weather cycles: real or imaginary. Ed Cambridge University Press. Cambridge. 207 p.

CHAMBERS, F.M., OGLE, M.I. BLACKFORD, J.J. (1999): «Paleoenvironmental evidence for solar forcing of Holocene climate: linkages to solar science». Progress in Physical Geography, 23 (2), $181-$ 204.

CREUS J. (1991): «Tendencia secular de la temperatura de mayo en el Pirineo Central (1302-1989)». Cuadernos de Geografía Física, 20-21, 41-49.

CREUS, J. (1996): «Evolución de la temperatura y precipitación anuales desde el año 1400 en el sector central de la depresión del Ebro». Lucas Mallada, 8, 9-27.

CREUS, J. (1998): «Variabilidad y estabilidad del clima español durante el último milenio. Estudio de fuentes dendrocronológicas». En Tecnología geográfica para el siglo XXI. Universidad de Barcelona, Barcelona (en prensa). 
CREUS, J., BEORLEGUI, M., FERNÁNDEZ, A. (1995): Reconstrucciones climáticas en Galicia durante las últimas centurias. Estudio Dendrocronológico. Ed. Presidencia Xunta de Galicia. La Coruña. 184 p.

CREUS, J., MARTÍNEZ, J, PUIGDEFÁBREGAS, J., (1980): «Fluctuaciones periódicas dentro de una dendrocronología pirenaica». Studin Oecologica, vol 1, 131-140.

CREUS, J. y SAZ, M.A. (1999): «Estudio de la variabilidad climática del último milenio a partir de series de temperatura y precipitación reconstruidas en el noreste español». En RASO J.M. y MARTÍN VIDE, J. (eds.): La climatología española en los albores del siglo XXI. Publicaciones de la Asociación Española de Climatología (AEC). Serie A, n. ${ }^{\circ}$ 1, 155-164.

CURRIE, R.G. (1991a): «Deterministic signals in tree rings from Tasmania, New Zealand, and South Africa». Annales Geophysicae, 8, 71-81.

CURRIE, R.G. (1991b): «Deterministic signals in tree rings from the Corn Belt Region». Annales Geophysicae, 9, 565-570.

CURRIE, R.G. (1991c): «Deterministic signals in tree rings from North America». International Journal of Climatology, 11, 861-876.

CURRIE, R.G. (1992): «Deterministic signals in tree rings from Europe». Annnles Geoplyysicae, 10, $241-253$.

CLAEVELAND, M.K., DUVICK, D.N., (1992): «Iowa climate reconstructed from tree rings, 16401982». Water Resources Research 28 (10), 2607-2615.

DAVIS, J. (1986): Statistics and data analysis in Geology. Ed. Willey and Sons. Singapur. 646 p.

DOUGLASS, A.E. (1933): «Evidences of cycles in tree ring records». Proceedings National Acndeny of Sciencies 19(3), 350-360.

ESSENWANGER, O.M. (1986): Elements of Statistical Analysis. En LANDSBERG, H.E. (ed): World Survey of Climatology, Volume 1B,. Ed.Elsevier. Londres.

LAMB, H.H. (1972): Climate: present, past and future. Mathuen \& Co. Londres.

LA MARCHE, V.C. (1974): «Paleoclimatic inferences from long tree ring records». Science, 183, 10431048 .

MITRA, K y DUTTA, S.N.: «18,6 year Luni-Solar nodal and 10-11 year solar signals in rainfall in India». International Journal of Climatology, 12, 839-851.

MANLEY, G. (1974): «Central England temperatures: monthly means 1659-1973». Quaternary Journal of the Royal Meteorological Society, 100, 389-405.

MITCHELL, J.M., STOCKTON, C.W., y MEKO, D.M. (1979): «Evidence of a 22 year rhythm of drought in the Western United States related to the Hale Solar Cycle since the $17^{\text {th }}$ centurys. En Solar-Terrestrial Influences on Weather E Climate, B.M. McCormac \& T.A. Seliga (eds), D. Reidel Publishing Co. Dordrecht. 
ROYER, J.F. y NICOLIS, C. (1994): «Chaos et Météorologie». La Météorologie, 8a serie, 5, 38-53.

SAZ, M.A. y CREUS, J. (1998): «Estudio dendroclimático de las rachas secas y húmedas en el sector central de la Depresión del Ebro». Geographicalia, 36, 159-172.

SAZ, M.A. y CREUS, J. (1999): "La variabilidad del clima español en el pasado: frecuencia de valores extremos de la temperatura y precipitación trimestrales reconstruidas desde el siglo XV». En RASO J.M. y MARTIN VIDE, J. (eds.): La climatología española en los albores del siglo XXI. Publicaciones de la Asociación Española de Climatología (AEC). Serie A, n. ${ }^{\circ}$ 1, 155-164.

SWAN, A.R.H. y SANDILANDS, M. (1995): Introduction to Geological Data Analysis. Ed. Blackwell Sciencie Ltd. Londres. 431 p. 